\title{
Relating Gribov-Zwanziger theory and Yang-Mills theory in Batalin-Vilkovisky formalism
}

\author{
Sudhaker Upadhyay* and Bhabani Prasad Mandal ${ }^{\dagger}$ \\ Department of Physics, \\ Banaras Hindu University, \\ Varanasi-221005, INDIA.
}

\begin{abstract}
We consider the BRST invariant Gribov-Zwanziger theory with appropriate horizon term in Batalin-Vilkovisky formalism. The usual infinitesimal BRST transformation is generalized by considering the parameter finite and field dependent. We show that such finite field dependent BRST transformation with suitable choice of finite parameter relates the generating functional of Gribov-Zwanziger theory to that of the Yang-Mills theory.
\end{abstract}

\section{INTRODUCTION}

In non-Abelian gauge theories even after gauge fixing the redundancy of gauge fields is not completely removed in certain gauges for large gauge fields (Gribov ambiguity) [1] . In order to resolve the Gribov ambiguity, Gribov and Zwanziger restrict the domain of integration in the functional integral to the so-called Gribov region $\Omega$ (defined in such a way that the Faddeev-Popov (FP) operator is strictly positive. i.e. $\Omega \equiv\left\{A_{\mu}^{a}, \partial_{\mu} A_{\mu}^{a}=\right.$ $\left.\left.0, \mathcal{M}^{a b}>0\right\}\right)$, whose boundary is known as the first Gribov horizon [2]. This has been achieved by adding a nonlocal term, commonly known as horizon term, to the YangMills (YM) action [2]. The non-Abelian gauge theories in those gauges contain so-called Gribov copies. Gribov copies play a crucial role in the infrared (IR) regime while it can be neglected in the perturbative ultraviolet (UV) regime [1, 2]. Such theories do not

\footnotetext{
* e-mail address: sudhakerupadhyay@gmail.com

† e-mail address: bhabani.mandal@gmail.com
} 
satisfy the Kugo-Ojima (KO) criterion for color confinement [3] since the presence of the nonlocal horizon term breaks the BRST invariance of the theory [4]. Recently, it has been shown that the BRST symmetry of the Gribov-Zwanziger (GZ) theory can be restored by introducing the auxiliary fields which explains the $\mathrm{KO}$ analysis [5].

In the present work we generalize such extended BRST transformation for GZ theory by allowing the parameter to be finite and field dependent following the method developed in the reference [6]. Such a generalized BRST (FFBRST) transformation is also a symmetry of the effective action. However, being finite in nature such a transformation does not leave the path integral measure as well as the generating functional invariant. We consider the Batalin-Vilkovisky (BV) formulation of this theory. For an appropriate choice of finite field dependent parameter we show that such a finite transformation relates the generating functional for GZ theory to the generating functional in YM theory in BV formulation.

The paper is organized as follows. In Sec. II we discuss briefly the GZ theory and extended BRST symmetry. Sec. III is devoted to the construction of FFBRST transformation. Connection of GZ theory and YM theory in BV formalism is established in Sec. IV. Last section is reserved for conclusions.

\section{PRELIMINARY: THE GZ THEORY}

The generating functional for standard GZ theory [2, 5, 7] reads as

$$
Z_{G Z}=\int[D \phi] e^{-S_{G Z}}, \quad S_{G Z}=S_{Y M}+S_{h},
$$

where the YM effective action $S_{Y M}$ in Landau gauge and local horizon term $S_{h}$ are written as

$$
\begin{aligned}
S_{Y M} & =\int d^{4} x\left[\frac{1}{4} F_{\mu \nu}^{a} F_{\mu \nu}^{a}+B^{a} \partial_{\mu} A_{\mu}^{a}+\bar{c}^{a} \partial_{\mu} \mathcal{D}_{\mu}^{a b} c^{b}\right], \\
S_{h} & =\int d^{4} x\left[\bar{\varphi}_{i}^{a} \partial_{\mu} \mathcal{D}_{\mu}^{a b} \varphi_{i}^{b}-\bar{\omega}_{i}^{a} \partial_{\mu} \mathcal{D}_{\mu}^{a b} \omega_{i}^{b}-g f^{a b c} \partial_{\mu} \bar{\omega}_{i}^{a} \mathcal{D}_{\mu}^{b d} c^{d} \varphi_{i}^{c}\right. \\
& \left.-\gamma^{2} g\left(f^{a b c} A_{\mu}^{a} \varphi_{\mu}^{b c}+f^{a b c} A_{\mu}^{a} \bar{\varphi}_{\mu}^{b c}+\frac{4}{g}\left(N^{2}-1\right) \gamma^{2}\right)\right] .
\end{aligned}
$$


The conventional BRST transformation for all the fields is given by

$$
\begin{aligned}
& \delta_{b} A_{\mu}^{a}=-\mathcal{D}_{\mu}^{a b} c^{b} \Lambda, \quad \delta_{b} c^{a}=\frac{1}{2} g f^{a b c} c^{b} c^{c} \Lambda, \quad \delta_{b} \bar{c}^{a}=B^{a} \Lambda, \quad \delta_{b} B^{a}=0, \\
& \delta_{b} \varphi_{i}^{a}=-\omega_{i}^{a} \Lambda, \quad \delta_{b} \omega_{i}^{a}=0, \quad \delta_{b} \bar{\omega}_{i}^{a}=\bar{\varphi}_{i}^{a} \Lambda, \quad \delta_{b} \varphi_{i}^{a}=0
\end{aligned}
$$

where $\Lambda$ is usual infinitesimal and global BRST parameter and index $i(\equiv \mu, a)$ is a composite index. Here we see that the BRST symmetry is broken softly for the GZ action [2], the breaking is due to the presence of $S_{h}$ term. To discuss the renormalizability of this theory the horizon term $S_{h}$ is extended by introducing 3 doublets of sources $\left(U_{\mu}^{a i}, M_{\mu}^{a i}\right),\left(V_{\mu}^{a i}, N_{\mu}^{a i}\right)$ and $\left(T_{\mu}^{a i}, R_{\mu}^{a i}\right)$, as

$$
\begin{aligned}
\Sigma_{\gamma} & =\int d^{4} x\left[-M_{\mu}^{a i} \mathcal{D}_{\mu}^{a b} \varphi^{b i}-g f^{a b c} U_{\mu}^{a i} \mathcal{D}_{\mu}^{b d} c^{d} \varphi^{c i}+U_{\mu}^{a i} \mathcal{D}_{\mu}^{a b} \omega^{b i}-N_{\mu}^{a i} \mathcal{D}_{\mu}^{a b} \bar{\omega}^{b i}\right. \\
& -V_{\mu}^{a i} \mathcal{D}_{\mu}^{a b} \bar{\varphi}^{b i}+g f^{a b c} V_{\mu}^{a i} \mathcal{D}_{\mu}^{b d} c^{d} \bar{\omega}^{c i}-M_{\mu}^{a i} V_{\mu}^{a i}+U_{\mu}^{a i} N_{\mu}^{a i}-g f^{a b c} R_{\mu}^{a i} \mathcal{D}_{\mu}^{b d} c^{d} \bar{\omega}^{c i} \\
& \left.+g f^{a b c} T_{\mu}^{a i} \mathcal{D}_{\mu}^{b d} c^{d} \bar{\varphi}^{c i}\right] .
\end{aligned}
$$

The sources $M_{\mu}^{a i}, V_{\mu}^{a i}, R_{\mu}^{a i}$ are commuting and the remaining three $U_{\mu}^{a i}, N_{\mu}^{a i}, T_{\mu}^{a i}$ are fermionic in nature. The BRST symmetry transformation for this extended sources are given as

$$
\delta_{b} U_{\mu}^{a i}=M_{\mu}^{a i} \Lambda, \delta_{b} V_{\mu}^{a i}=-N_{\mu}^{a i} \Lambda, \delta_{b} T_{\mu}^{a i}=-R_{\mu}^{a i} \Lambda, \delta_{b} M_{\mu}^{a i}=\delta_{b} N_{\mu}^{a i}=\delta_{b} R_{\mu}^{a i}=0 .
$$

To make the extended theory reminiscent with original theory (2.1), at the end, sources are equated to have following physical values [5]:

$$
\left.U_{\mu}^{a i}\right|_{p h y s}=\left.N_{\mu}^{a i}\right|_{p h y s}=\left.T_{\mu}^{a i}\right|_{p h y s}=0,\left.M_{\mu \nu}^{a b}\right|_{p h y s}=\left.V_{\mu \nu}^{a b}\right|_{p h y s}=\left.R_{\mu \nu}^{a b}\right|_{p h y s}=\gamma^{2} \delta^{a b} \delta_{\mu \nu},
$$

such that $\left.\Sigma_{\gamma}\right|_{p h y s}=S_{h}$.

\section{CONSTRUCTION OF FFBRST TRANSFORMATION IN EUCLIDEAN SPACE}

The properties of BRST transformation do not depend on whether the parameter $\Lambda$ is (i) finite or infinitesimal, (ii) field dependent or not, as long as it is anticommuting 
in nature. Keeping this in mind, Joglekar and Mandal introduced finite field dependent BRST transformation [6], which has found many applications in gauge field theories [611]. Following these observations we make the parameter $\Lambda$ given in Eqs. (2.3) and (2.5) finite and field dependent without losing its properties. To construct the FFBRST transformations we start with making the infinitesimal parameter field dependent by introducing a parameter $\kappa(0 \leq \kappa \leq 1)$ and making all the fields, $\phi(x, \kappa), \kappa$ dependent such that $\phi(x, \kappa=0)=\phi(x)$ and $\phi(x, \kappa=1)=\phi^{\prime}(x)$, the transformed field.

The usual infinitesimal BRST transformations, thus can be written generically as

$$
d \phi(x, \kappa)=\delta_{b}[\phi(x, \kappa)] \Theta^{\prime}[\phi(x, \kappa)] d \kappa,
$$

where the $\Theta^{\prime}[\phi(x, \kappa)] d \kappa$ is the infinitesimal but field dependent parameter. The generalized BRST transformations with the finite field dependent parameter then can be constructed by integrating such infinitesimal transformations from $\kappa=0$ to $\kappa=1$, to obtain

$$
\phi^{\prime} \equiv \phi(x, \kappa=1)=\phi(x, \kappa=0)+\delta_{b}[\phi(x)] \Theta[\phi(x)]
$$

where $\Theta[\phi(x)]=\int_{0}^{1} d \kappa^{\prime} \Theta^{\prime}\left[\phi\left(x, \kappa^{\prime}\right)\right]$, is the finite field dependent parameter. Following this method, the BRST transformation, in Eqs. (2.3) and (2.5), is generalized such that the parameter is finite and field dependent. Such generalized BRST transformation is symmetry of the effective action. However, the path integral measure $[D \phi]$ in Eq. (2.1) is not invariant under such transformation as the BRST parameter is finite and field dependent. The Jacobian of the path integral measure (defined through Eq. $D \phi^{\prime}=$ $J(\kappa) D \phi)$ in Euclidean space for such transformations can be evaluated for some particular choice of the finite field dependent parameter, $\Theta[\phi(x)]$. Such Jacobian contribution can be replaced (within the functional integral) by $\exp \left[-S_{1}[\phi(x, \kappa)]\right]$, iff the following condition is satisfied [7]

$$
\int D \phi(x)\left[\frac{1}{J} \frac{d J}{d \kappa}+\frac{d S_{1}[\phi(x, \kappa)]}{d \kappa}\right] \exp \left[-\left(S_{G Z}+S_{1}\right)\right]=0,
$$

where $S_{1}[\phi]$ is some local functional of fields. The infinitesimal change in the $J(\kappa)$ can be calculated using the equation [6]

$$
\frac{1}{J} \frac{d J}{d \kappa}=-\int d^{4} x\left[\Sigma_{\phi}\left\{ \pm \delta_{b} \phi(x, \kappa) \frac{\partial \Theta^{\prime}[\phi(x, \kappa)]}{\partial \phi(x, \kappa)}\right\}\right]
$$


where \pm sign refers to whether $\phi$ is a bosonic or a fermionic field.

The FFBRST transformation corresponding to the BRST transformation given in Eqs. (2.3) and (2.5) is then written as

$$
\begin{aligned}
& \delta_{b} A_{\mu}^{a}=-\mathcal{D}_{\mu}^{a b} c^{b} \Theta ; \delta_{b} c^{a}=\frac{1}{2} g f^{a b c} c^{b} c^{c} \Theta ; \delta_{b} \bar{c}^{a}=B^{a} \Theta ; \delta_{b} B^{a}=0 ; \\
& \delta_{b} \omega_{i}^{a}=0 ; \delta_{b} \varphi_{i}^{a}=-\omega_{i}^{a} \Theta ; \delta_{b} \bar{\omega}_{i}^{a}=\bar{\varphi}_{i}^{a} \Theta ; \delta_{b} \varphi_{i}^{a}=0 ; \quad \delta_{b} U_{\mu}^{a i}=M_{\mu}^{a i} \Theta ; \\
& \delta_{b} T_{\mu}^{a i}=-R_{\mu}^{a i} \Theta ; \delta_{b} V_{\mu}^{a i}=-N_{\mu}^{a i} \Theta ; \delta_{b} M_{\mu}^{a i}=\delta_{b} N_{\mu}^{a i}=\delta_{b} R_{\mu}^{a i}=0,
\end{aligned}
$$

where $\Theta$ is finite, field dependent, anticommuting and explicit space-time independent parameter.

\section{CONNECTING GZ THEORY AND YM THEORY IN BV FORMALISM}

The generating functional of YM theory in the BV formulation can be written by introducing antifields $\phi^{\star}$ corresponding to the all fields $\phi$ with opposite statistics as,

$$
Z_{Y M}=\int[D \phi] e^{-\int d^{4} x\left\{\frac{1}{4} F_{\mu \nu}^{a} F_{\mu \nu}^{a}+A_{\mu}^{a \star} \mathcal{D}_{\mu}^{a b} c^{b}+\bar{c}^{a \star} B^{a}\right\}} .
$$

This can further be written in compact form as

$$
Z_{Y M}=\int[D \phi] e^{-\left[W_{\Psi_{1}}\left(\phi, \phi^{\star}\right)\right]}
$$

where the expression for gauge-fixing fermion is given as $\Psi_{1}=\int d^{4} x\left[\bar{c}^{a} \partial_{\mu} A_{\mu}^{a}\right]$. The generating functional does not depend on the choice of gauge-fixing fermion [12]. The extended quantum action, $W_{\Psi_{1}}\left(\phi, \phi^{\star}\right)$, satisfies certain rich mathematical relation called quantum master equation [13], given by

$$
\Delta e^{-W_{\Psi_{1}}\left[\phi, \phi^{\star}\right]}=0 \text { with } \Delta \equiv \frac{\partial_{r}}{\partial \phi} \frac{\partial_{r}}{\partial \phi^{\star}}(-1)^{\epsilon+1} .
$$

The antifields can be evaluated from $\Psi_{1}$ as

$$
A_{\mu}^{a \star}=\frac{\delta \Psi_{1}}{\delta A_{\mu}^{a}}=-\partial_{\mu} \bar{c}^{a}, \quad \bar{c}^{a \star}=\frac{\delta \Psi_{1}}{\delta \bar{c}^{a}}=\partial_{\mu} A_{\mu}^{a}, \quad B^{a \star}=\frac{\delta \Psi_{1}}{\delta B^{a}}=0 .
$$


Similarly, the generating functional of GZ theory in BV formulation can be written as,

$$
\begin{aligned}
Z_{G Z} & =\int[D \phi] \exp \left[-\int d^{4} x\left\{\frac{1}{4} F_{\mu \nu}^{a} F_{\mu \nu}^{a}+A_{\mu}^{a \star} \mathcal{D}_{\mu}^{a b} c^{b}+\bar{c}^{a \star} B^{a}-\varphi_{i}^{b \star} \omega_{i}^{b}\right.\right. \\
& \left.\left.++\bar{\varphi}_{i}^{a} \bar{\omega}_{i}^{a \star}+U_{\mu}^{a i \star} M_{\mu}^{a i}-V_{\mu}^{a i \star} N_{\mu}^{a i}-T_{\mu}^{a i \star} R_{\mu}^{a i}\right\}\right] .
\end{aligned}
$$

This can further be written in compact form using gauge-fixed fermion $\left(\Psi_{2}\right)$ as

$$
\begin{aligned}
Z_{G Z} & =\int[D \phi] e^{-\left[W_{\Psi_{2}}\left(\phi, \phi^{\star}\right)\right]}, \Psi_{2}=\int d^{4} x\left[\bar{c}^{a} \partial_{\mu} A_{\mu}^{a}+\bar{\omega}_{i}^{a} \partial_{\mu} \mathcal{D}_{\mu}^{a b} \varphi_{i}^{b}\right. \\
& \left.-U_{\mu}^{a i} \mathcal{D}_{\mu}^{a b} \varphi^{b i}-V_{\mu}^{a i} \mathcal{D}_{\mu}^{a b} \bar{\omega}^{b i}-U_{\mu}^{a i} V_{\mu}^{a i}+g f^{a b c} T_{\mu}^{a i} \mathcal{D}_{\mu}^{b d} c^{d} \bar{\omega}^{c i}\right] .
\end{aligned}
$$

The antifields are obtained from $\Psi_{2}$ as

$$
\begin{array}{r}
A_{\mu}^{a \star}=-\partial_{\mu} \bar{c}^{a}-g f^{a b c} \partial_{\mu} \omega^{c i} \varphi^{b i}-g f^{a b c} U_{\mu}^{c i} \varphi^{b i}-g f^{a b c} V_{\mu}^{c i} \bar{\omega}^{b i}, \quad \bar{c}^{a \star}=\partial_{\mu} A_{\mu}^{a}, \\
U_{\mu}^{a i \star}=-\mathcal{D}_{\mu}^{a b} \varphi^{b i}-V_{\mu}^{a i}, \quad \bar{\omega}_{i}^{a \star}=\partial_{\mu} \mathcal{D}_{\mu}^{a b} \varphi_{i}^{b}-V_{\mu i}^{b} \mathcal{D}_{\mu}^{b a}+g f^{a b c} T_{\mu i}^{b} \mathcal{D}_{\mu}^{c d} c^{d}, \\
V_{\mu}^{a i \star}=-\mathcal{D}_{\mu}^{a b} \bar{\omega}^{b i}-U_{\mu}^{a i}, T_{\mu}^{a i \star}=g f^{a b c} \mathcal{D}_{\mu}^{b d} c^{d} \bar{\omega}^{c i}, \varphi_{i}^{b \star}=\bar{\omega}_{i}^{a} \partial_{\mu} \mathcal{D}_{\mu}^{a b}-U_{\mu i}^{a} \mathcal{D}_{\mu}^{a b} .
\end{array}
$$

To connect these two theories we construct following finite field dependent parameter $\Theta[\phi]$

$$
\Theta[\phi]=\int_{0}^{1} d \kappa \int d^{4} x\left[\varphi_{i}^{b \star} \varphi_{i}^{b}+\bar{\omega}_{i}^{b \star} \bar{\omega}_{i}^{b}+V_{\mu}^{a i \star} V_{\mu}^{a i}\right] .
$$

The Jacobian of path integral measure in the generating functional (2.1) for the FFBRST with this parameter can be replaced by $e^{-S_{1}}$ iff condition (3.3) is satisfied. To find $S_{1}$ we start with an ansatz for $S_{1}$ as

$$
S_{1}=\int d^{4} x\left[\chi_{1} \varphi_{i}^{b \star} \omega_{i}^{b}+\chi_{2} \bar{\varphi}_{i}^{a} \bar{\omega}_{i}^{a \star}+\chi_{3} U_{\mu}^{a i \star} M_{\mu}^{a i}+\chi_{4} V_{\mu}^{a i \star} N_{\mu}^{a i}+\chi_{5} T_{\mu}^{a i \star} R_{\mu}^{a i}\right] .
$$

where $\chi_{j}(\kappa)(j=1,2, . ., 5)$ are arbitrary but $\kappa$-dependent constants and satisfy following initial conditions $\chi_{j}(\kappa=0)=0$. These constants are calculated using Eq. (3.3) subjected to the initial condition to find the $S_{1}$ as

$$
S_{1}=\int d^{4} x\left[+\kappa \varphi_{i}^{b \star} \omega_{i}^{b}-\kappa \bar{\varphi}_{i}^{a} \bar{\omega}_{i}^{a \star}-\kappa U_{\mu}^{a i \star} M_{\mu}^{a i}+\kappa V_{\mu}^{a i \star} N_{\mu}^{a i}+\kappa T_{\mu}^{a i \star} R_{\mu}^{a i}\right] .
$$

By adding $S_{1}(\kappa=1)$ to $S_{G Z}$, we get $S_{G Z}+S_{1}(\kappa=1)=S_{Y M}$. Hence,

$$
Z_{G Z}\left(\equiv \int[D \phi] e^{-W_{\Psi_{2}}}\right)-\stackrel{F F B R S T}{\longrightarrow} Z_{Y M}\left(\equiv \int[D \phi] e^{-W_{\Psi_{1}}\left(\phi, \phi^{\star}\right)}\right)
$$

Thus, using FFBRST transformation we connect the GZ theory to YM theory in BV formulation. 


\section{CONCLUSION}

The KO criterion for color confinement in a manifestly covariant gauge is not satisfied in GZ theory due to the presence of the horizon term which breaks the usual BRST symmetry. However this theory is extended to restore the BRST symmetry and hence the KO analysis is satisfied for color confinement. This BRST symmetry is generalized by allowing the transformation parameter finite and field dependent in the context of BV formulation. Such FFBRST transformation is also symmetry of the effective action $S_{G Z}$ but not of the generating functional $Z_{G Z}$. We have shown that the FFBRST with an appropriate choice of finite field dependent parameter relates GZ theory with a correct horizon term to the YM theory in the BV formulation. Thus the theory free from Gribov copies has shown to be related to a theory with Gribov copies (i.e. YM theory) through FFBRST transformation in the BV formulation. The nontrivial Jacobian of path integral measure in $Z_{G Z}$ is responsible for this important connection.

\section{Acknowledgment}

One of us (SU) acknowledge the financial assistance from DST, India under ITS scheme (grant No. R/ITS/3443/2011-2012) and organizers of "8 ${ }^{\text {th }}$ International Conference on Progress in Theoretical Physics" Constantine, Algeria.

[1] V. N. Gribov, Nucl. Phys. B 139, 1 (1978).

[2] D.Zwanziger, Nucl. Phys. B 323, 513 (1989).

[3] T. Kugo and I. Ojima, Suppl. Prog. Theor. Phys. 66, 1 (1979).

[4] D. Dudal et al, Phys. Rev. D 78065047 (2008).

[5] D. Dudal et al Eur. Phys. J. C 68, 283 (2010).

[6] S. D. Joglekar and B. P. Mandal, Phys. Rev. D 51, 1919 (1995).

[7] S. Upadhyay and B. P. Mandal, Euro. Phys. Lett. 93, 31001 (2011).

[8] R. Banerjee and B. P. Mandal, Phys. Lett. B27 488 (2000). 
[9] B. P. Mandal, S. K. Rai and S. Upadhyay, Euro. Phys. Lett. 92, 21001 (2010).

[10] S. Upadhyay, S. K. Rai and B. P. Mandal, J. Math. Phys. 52, 022301,(2011).

[11] S. Upadhyay and B. P. Mandal, Mod. Phys. Lett. A 25, 3347 (2010).

[12] M. Henneaux and C. Teitelboim, Quantization of gauge systems (Princeton, USA: Univ. Press, 1992).

[13] S. Weinberg, The quantum theory of fields, Vol-II: Modern applications (Cambridge, UK Univ. Press, 1996). 